Corps et culture

Numéro 3 | 1998

Sport et lien social

\title{
Sport-aventure et lien social : l'exemple du Raid Passion Hérault
}

\section{Gisèle Lacroix}

\section{(2) OpenEdition}

1 Journals

Édition électronique

URL : http://journals.openedition.org/corpsetculture/439

DOI : 10.4000/corpsetculture.439

ISSN : $1777-5337$

Éditeur

Association Corps et Culture

Édition imprimée

Date de publication : 1 juin 1998

ISSN : 1268-5631

Référence électronique

Gisèle Lacroix, « Sport-aventure et lien social : l'exemple du Raid Passion Hérault », Corps et culture [En ligne], Numéro 3 | 1998, mis en ligne le 04 mai 2007, consulté le 08 septembre 2020. URL : http:// journals.openedition.org/corpsetculture/439; DOI : https://doi.org/10.4000/corpsetculture.439

Ce document a été généré automatiquement le 8 septembre 2020

(c) tous droits réservés 


\title{
Sport-aventure et lien social : l'exemple du Raid Passion Hérault
}

\author{
Gisèle Lacroix
}

1 Le sport a toujours été créateur de spectacles qui se sont structurés pour certains en grands événements ayant leur périodicité. L'évolution récente montre que leur nombre et leur fréquence se sont quantitativement accrus pendant que leurs formes se différenciaient en faisant varier à l'infini leurs paramètres : organisateurs, partenaires, activités pratiquées, objectifs, publics ciblés, lieux et durée....

2 Les formes d'événements sont ici un analyseur de l'évolution des pratiques corporelles : du modèle sportif compétitif contrôlé par les instances fédérales à celui plus participatif et informel des pratiques physiques de loisir. Elles permettent d'interroger les «nouvelles » formes d'être ensemble que l'individu construit au sein d'un autre type d'organisation.

3 Le Raid Passion Hérault pris en exemple ici, est un événement qui appartient au registre des épreuves de sport-aventure dans leurs modalités « douces ». Optant pour une mise à distance de certaines dimensions du sport traditionnel et privilégiant « la fête ", il fait l'hypothèse de favoriser un mode de sociabilité fondé davantage sur le partage, la convivialité et où l'expression de soi se conjugue avec le sentiment d'appartenance à une équipe, une ville..... Pour cela, il joue sur les structures organisationnelles formelles qui, par la marge de liberté laissée aux acteurs, conditionnent aussi l'informel et l'imaginaire véhiculé par l'événement.

4 Le projet est donc ici de mettre en rapport l'offre faite dans la production d'un événement conçu à partir d'une analyse de la demande, avec le vécu des participants, en tentant de qualifier les modes de sociabilité dont il permet l'expression. Pour cela, le regard se veut attentif au « sensible » défini comme «ce qui nous affecte et retentit en nous »; ou encore le niveau où se produit « la conjonction la plus élémentaire et la plus énigmatique (la plus admirable) du sens des sens » (SANSOT P., cité par LE POGAM, Y, 1995, p. 109). Tentative modeste pour saisir les potentialités émotionnelles que ces épreuves exacerbent par le cadre, la présence du groupe, le défi physique, produisant 
ce mélange de réel et d'irréel : ambiance particulière pour une part explicative de leur engouement actuel.

5 En arrière-fond, cette expérimentation interroge le statut de l'individu: est-il acteur (auto-déterminé ?) ou consommateur (aliéné ?) ; elle s'inscrit dans un débat où la « rationalité instrumentale » et la « rationalité humaniste » s'opposent encore par les visions de l'individu privilégiant la soumission à l'ordre productif et rentable ou bien l'expression de la souveraineté et du différentialisme dans des pratiques transgressives (LE POGAM, Y., 1998).

La conception du Raid Passion HéraultLe sport-aventure

6 A la suite des grands raids créés dans les années 80 (comme le Paris-Dakar, le CamelTrophy, Harricana, le Raid Ivoire....) sont nées des épreuves associant différentes disciplines (le Raid Gauloises en est le symbole) qui mettent en scène des activités physiques de pleine nature, écologiques au sens de "non mécaniques". Baptisées "sport-aventure », ces épreuves par le mariage des deux termes révèlent une double transformation. Il y a en effet, à la fois un " usage non sportif du sport » (par l'absence de référence aux espaces, aux temps et aux catégories sportives habituelles....) et une réintroduction des dimensions de la compétition sportive traditionnelle (chronométrage, règlement, classement...) ; à la fois aventure par l'imprévisibilité et le risque (réel ou imaginaire) mais celle-ci est organisée et «sportivisée », perdant par là même ces caractéristiques d'inconnu ${ }^{1}$.

Depuis leur apparition, ces manifestations ont remporté un franc succès et la logique économique, pour répondre à une demande multiforme, les a décliné de l'aventure élitiste à l'aventure de masse, jusqu'au tourisme d'aventure : elles sont alors de moins en moins risquées et éprouvantes, de moins en moins techniques et sportives, donc de plus en plus «douces » et accessibles à tous. Elles sont aussi : proches ou lointaines, conviviales ou rivalitaires suivant les paramètres mis en avant par l'organisation, ....mais de toute façon hors des sentiers battus par le sportif ou le touriste " ordinaire ".

La conception et la création du Raid Passion Hérault s'inscrivent dans cette évolution des pratiques. C'est une épreuve associant différentes activités, essentiellement de pleine nature (course à pied, VTT avec ou sans orientation, canoë, tir à l'arc....). Elle dure 3 jours et se déroule le long du fleuve Hérault. Dans la déclinaison des épreuves, elle se positionne comme accessible à tous, intégrant la dimension culturelle et touristique, sans se départir d'un certain engagement physique.

Un produit « innovant »

La société postmoderne se distingue par de "nouvelles procédures inséparables de nouvelles finalités et légitimités sociales : valeurs hédonistes, respect des différences, culte de la libération personnelle, de la décontraction, de l'humour et de la sincérité, psychologisme, expression libre.... » (LIPOVETSKY, 1983). Dix années de regard sur les "nouvelles pratiques " ${ }^{2}$, à la lumière des théories postmodernes montrent que ces valeurs narcissiques se traduisent dans les pratiques corporelles par la recherche d'activités auto-déterminées, dans des lieux ouverts, sans modèle hégémonique et laissant libre cours aux imaginaires. C'est en cela qu'elles s'inscrivent (du moins à leur apparition) en rupture avec le modèle sportif "traditionnel" et les valeurs qu'il véhicule (effort, hiérarchie, sélection, rentabilité).

10 Pour être innovant, c'est-à-dire susceptible de laisser s'instaurer une autre façon d'être soi et d'être ensemble, le Raid Passion Hérault se dote de caractéristiques instaurant 
une "cassure avec la logique sportive élitiste", favorisant l'autodétermination, l'ouverture (au milieu, aux autres) et jouant avec un imaginaire actuellement valorisé. Une distanciation du modèle sportif compétitif

11 Le Raid Passion Hérault veut permettre une "entrée non sportive dans le sport » (au sens de P. YONNET, 1993), c'est-à-dire sans barrière techniciste ou morphologique. Ceci se module par le choix d'activités accessibles à tous (vélo, course à pied, canoë) dans un milieu facilitant qui ne demande ni un investissement énergétique, ni un engagement corporel important. La codification ou la réglementation seront réduites au minimum. L'aléa des épreuves d'orientation (pour des non-spécialistes) relativise encore l'investissement énergétique (gagner difficilement trois minutes sur un parcours..... pour ensuite chercher une balise une heure.....)

12 Les activités sportives sont décloisonnées (contrairement au fonctionnement fédéral) par le mélange ou l'invention de pratiques (par exemple l'escalabranche) ou encore par l'association à des «défis intellectuels» (questionnaire culturel sur la région traversée.......).

13 Le collectif prime sur l'individuel : c'est la performance du groupe qui est prise en compte, ce qui privilégie l'entraide au détriment de la valorisation individuelle, favorise la dimension ludique et conviviale tout en relativisant le classement. L'inscription donne droit à une dotation d'équipement identique pour tous et les rituels de consécration (le classement par étape......) vont dans le sens de la récompense de tous. En variant les critères (l'équipe la plus «fair-play ", malchanceuse......) ou par tirage au sort, l'organisation est attentive à gratifier chaque équipe au cours du raid. $\mathrm{Si}$ le classement final couronne les équipes les plus performantes ${ }^{3}$, les lots sont sans grand décalage avec ceux attribués sur d'autres critères.

Dans ce cadre, les normes qui fondent la structure de l'équipe sont très importantes. Plus l'équipe est nombreuse (six personnes), plus il est difficile qu'elle soit « homogène » et donc performante, ce qui profite à "l'esprit d'équipe ». La présence des femmes et leur nombre (deux « minimum » par équipe) accentue la distanciation au sport : elles sont encore (statistiquement) moins impliquées dans la compétition, moins axées sur le résultat de la pratique, donnant plus d'importance à sa forme participative et aux relations qu'elle suscite (DAVISSE, LOUVEAU, 1991).

Autant d'éléments qui « marquent la distance » avec le modèle compétitif traditionnel, et l'organisation souhaite que puisse se côtoyer différentes façons de pratiquer, que chacun s'y exprime pleinement sans exclusion formelle ou informelle liée à la reconnaissance d'un modèle technique, performant, individualiste.

L'imaginaire véhiculé par l'événement

16 L'hypothèse $e^{4}$ selon laquelle l'innovation tient davantage à l'imaginaire véhiculé, à la "façon de pratiquer" qu'à la pratique elle-même, sert d'ancrage théorique à la création d'une épreuve innovante. Elle se traduit dans le Raid Passion Hérault par la recherche d'une symbolique forte et le respect de caractéristiques ménageant «l'esprit de liberté ".

Etant une épreuve de sport-aventure, le Raid Passion Hérault s'inscrit dans l'imaginaire que suscite actuellement l'aventure. L'idéologie de la nature « bonne et nourricière », le retour aux sources comme réconciliation avec une corporéité préexistante à la société industrielle, et le retour au corps comme révélation de soi (VIGARELLO, 1982) sont actifs dans les représentations des participants. De même, le risque et l'inconnu (plus 
imaginaires que réels) associés à ces pratiques, alimentent l'incontournable ordalie moderne d'un individu qui n'a plus que lui-même comme référence et redonne un sens à sa vie dans l'échange symbolique avec la mort (LE BRETON, 1991). Ces épreuves forgent l'image "des nouveaux héros » : baroudeurs, passionnés et jusqu'au-boutistes qui doivent leur survie tant à leurs capacités physiques qu'à leur endurance morale, et reviennent régénérés de l'épreuve qu'ils se sont imposés à eux-mêmes, «libération qu'on s'impose à soi au nom de soi » (EHRENBERG, 1991, p. 181).

Plus spécifiquement, le Raid Passion Hérault met l'accent sur une symbolique fédératrice qui passe par le fleuve Hérault, d'où le premier sous-titre du raid: « de la source à la mer....au fil de l'Hérault ». Il renvoie à la symbolique de l'eau comme élément vital (connotation écologique), comme dynamique : l'eau bouge, parcourt du chemin ; et comme synergie : le fleuve est un trait d'union, un fil conducteur fédérateur des énergies. Trait d'union géographique reliant la montagne et la mer ; trait d'union culturel par l'histoire du fleuve ; trait d'union économique notamment par le tourisme, il devient aussi un trait d'union relationnel fédérant les ressources humaines autour d'un projet. Cette symbolique est en accord avec la volonté de l'organisation d'ancrer le raid dans le développement local, d'impliquer les acteurs (contrairement à l'image négative de certains raids qui traversent un pays sans le connaître, ni rien lui apporter). Le nom du raid porte bien sûr celui du fleuve et tente de capter l'air du temps en adoptant le terme "Passion» qui renvoie aux valeurs, esthétiques, émotionnelles autant qu'à l'humeur anti-rationnaliste.

Pour être plus près de l'image des «nouvelles pratiques » qui se désignent comme « libres » et « en marge ", l'organisation réaménage l'espace, le temps, les structures et fait en sorte de donner libre cours à l'imaginaire de liberté. Les contraintes spatiales sont réduites au maximum : un départ, une arrivée, quelques points de passages obligés (changement de matériel, point d'assistance, ravitaillement....). L'espace appartient à l'équipe qui en use à sa guise : elle choisit son itinéraire, sa distance (hors des espaces codifiés, standardisés). Le choix du site (son immensité déserte, son caractère sauvage......) renforce l'impression de liberté et le sentiment de toute puissance qu'il génère. Cette liberté dans l'espace se complète d'une liberté dans le temps (en dehors de calendriers et de durées d'épreuves programmés à l'avance). Celle-ci s'inscrit dans la marge laissée entre les premiers et les derniers pour que la logique de l'épreuve ne soit pas calibrée sur la rapidité, la rentabilité des meilleurs mais sur le projet de l'équipe, l'usage du temps choisi par chacune (en fonction de son niveau, de ses objectifs, de sa manière de pratiquer). La préservation d'un espace-temps peu contraignant donne une autre liberté : celle de choisir son degré d'investissement physique (et psychique) : de " la défonce pure et dure » à une participation plus « cool et décontractée ». L'essentiel étant que l'organisation n'exclut ni les uns, ni les autres, tout en leur donnant des conditions égales de confort et d'encadrement. La volonté de liberté s'exprime également dans le rapport à l'organisation. Celle-ci se fait discrète pour favoriser l'autonomie....mais très présente pour le confort, la sécurité. Elle cherche à minimiser les contraintes formelles et informelles pour être flexible et adaptable à toute demande (un « libre service » pour le plaisir du pratiquant). La liberté dans le rapport aux autres se traduit dans le mode de constitution des équipes: pour vivre cette aventure, l'individu choisit ses pairs par affinité selon la logique du réseau.

La volonté festive affichée est la dernière touche d'innovation. La fête est une dimension phénoménologique qui renouvelle la relation de l'individu à lui-même, aux 
autres, au milieu..... et au sport. Elle renvoie à l'effervescence, à l'excès, au désordre. Elle est une transgression du modèle sportif: à l'abstinence, le rentable, l'investissement capitalisé s'opposent la générosité, le futile, la gratuité. L'idée est que la fête renoue avec le sport (elle lui était consubstantielle à son origine) qu'elle soit un vécu pour tous et non uniquement un spectacle : «la fête pour les hôtes comme pour les participants » (slogan de la plaquette du raid). Le déguisement obligatoire lors d'une épreuve est un pari : les concurrents vont-ils jouer le jeu? Il a aussi une fonction transgressive par rapport à la codification poussée de la tenue sportive en compétition faites de normes fédérales et économiques (les emplacements publicitaires). Dans cet objectif festif, l'accueil, l'ambiance, tout l'environnement physique et relationnel ( «le hors-épreuves ») sont aussi importants que les épreuves elles-mêmes.

21 "De la source à la mer... au fil de l'Hérault », le raid adopte comme deuxième soustitre: «Le sport, l'aventure, la fête ». La confrontation de la volonté organisatrice et des structures mises en place avec l'usage qui en est fait par les participants, suppose de sonder leurs expériences vécues....

Le vécu des participants

22 L'objet est d'éclairer les formes de lien social engendrées par ce type d'événement, celui-ci étant entendu comme l'expression, la participation de l'individu, et comme l'appropriation de l'événement par le projet du groupe et les relations qui se tissent.

L'image du Raid Passion Hérault

Une des questions invite les raiders à choisir le terme qui leur semble le mieux qualifier le raid et leur investissement, parmi : « le sport, l'aventure, la fête ».

Majoritairement, c'est l'aventure qui l'emporte mais avec des significations diversifiées. Elle est une rupture avec le quotidien, le travail, parce que l'aventure, c'est découvrir des espaces ( $\mathrm{ni}$ un stade, ni une piscine»), des paysages ("peu fréquentés»), «être une journée complètement dans la nature sans voir une maison, une route, une voiture ». C'est encore "partir », "faire ce que l'on a jamais fait» (l'inconnu relatif 5 ), avec l'idée de gratuité "arriver au bout et ne rien gagner». L'aventure est étroitement associée aux activités de pleine nature, par leur pratique peu fréquente, par les lieux inhabituels et sauvages qui supposent une compétence valorisée : «la débrouille " quelles que soient les circonstances («aimer bartasser en groupe »). La course d'orientation est la plus représentative de l'aventure : « la C.O., ça fait raid, avec la carte....chercher....se perdre sur le plateau...». Quand l'aventure est associée au sport, il est défini comme «le défi physique» pour "aller plus loin....chercher ses limites ». Plus ponctuellement, elle est vue comme « le plaisir d'être en groupe ", de faire un projet ensemble.

Une fois seulement, le Raid Passion Hérault est identifié à la fête seule pour la convivialité, la rencontre des autres équipes, et une autre associée au défi physique: « faire du sport et faire la fête ». Le sport seul est cité une fois, évoquant la difficulté des épreuves.

Interrogés sur les moments forts, les participants les traduisent surtout à travers le sport comme "défonce physique » et comme épreuve : «les montées insurmontables du premier jour »; mais aussi à la beauté du paysage (« la descente de l'Aigoual »), au mélange sport et fête "dur pendant les épreuves, cool le soir", et enfin à l'équipe : «s'encourager, tenir compte des autres». Ils sont aussi ceux des départs collectifs, 
moment de rassemblements intenses en émotion, rites d'agrégation qui recèlent une puissance affective toujours renouvelée.

Ces représentations du Raid Passion Hérault se recoupent avec les objectifs énoncés par rapport à soi : découvrir une région, des activités nouvelles, «aller au bout de soimême", "en baver» ou encore "s'amuser» et "transpirer avec les autres". L'aventure est donc prégnante, étroitement associée au sport comme défi, la fête est peu évoquée.

Les formes de l'être ensemble

28 La « cassure » avec le modèle sportif traditionnel est parfaitement perçue, approuvée et consommée («je ne suis pas là pour faire une performance»), la variété et l'enchaînement de pratiques, l'opportunité d'en découvrir de nouvelles, sont autant d'éléments perçus comme attractifs. La pratique en équipe, hautement appréciée, renouvelle les objectifs individuels et collectifs, les relations et les modalités d'affrontement.

29 La présence des femmes est effectivement une variable fondamentale de distanciation à la performance entendue au sens traditionnel, et elle renouvelle les rapports à l'intérieur de l'équipe. Si ELLES y voient une chance de pouvoir participer car sans l'obligation « il n'y aurait que des mecs »; EUX apprécient « la complémentarité » et se sentent même soulagés d'être dispensés de « se tirer dessus » dans " une compétition à outrance ». La mixité est vécue comme égalisant les équipes et régulatrice: "c'est ELLES qui donnent le ton "; elle amène un changement qualitatif dans les motivations et les relations. Pour EUX, l'objectif passe de la performance à la solidarité et l'entraide, quand ELLES sont stimulées et montrent que «l'effort ne leur fait pas peur». Des variations existent d'une équipe à l'autre : celles qui veulent « figurer honorablement » sont aussi les plus âgées et axées sur la convivialité ; par contre, quand les plus jeunes veulent « tenir leur place », la " pression » ressentie par les femmes est plus forte : elles ont "peur de ne pas suivre" mais espèrent «que les hommes apprendront à les considérer, même si les remarques fusent ». Pendant ce temps, dans l'équipe en tête, ILS apprécient «leurs féminines qui assurent » quand ELLES se sentent «entourées comme des reines ». L'âge, le niveau et l'homogénéité de l'équipe influencent l'objectif collectif en terme de résultat et modulent le ressenti de la mixité; mais pour tous, elle est vécue dans « une bonne ambiance ».

La notion de performance n'en est pas moins présente, mais sa signification devient autre et se lit dans les objectifs annoncés par rapport à soi et par rapport à l'équipe. Pour soi, il s'agit de "suivre " ou "d'encourager les autres", de ne pas se blesser, faiblir ou être ridicule. C'est aussi «tenir, aller au bout de soi-même, faire le mieux possible»; et ce, au service de la performance de l'équipe : "être dans les cinq premières » revient souvent ou «terminer honorablement». D'autres indicateurs confirment ce report du modèle compétitif individuel sur le collectif. Dans les réunions de chef d'équipes, les doléances concernent essentiellement les problèmes de matériels ou de balisage qui ont fait perdre du temps....donc des places dans le classement. Dans la cassette-vidéo tournée pendant le raid, qui interrogent «sur le vif» des équipes à l'arrivée, nombreuses sont les personnes qui traduisent leurs impressions en relation avec leur classement du jour et leurs espoirs pour les jours suivants ${ }^{7}$. Pourtant lors de la troisième journée, les conséquences du parcours VTT truffé de clous par la mauvaise humeur probable des chasseurs (environ 200 crevaisons en 3 heures) sont très bien 
acceptées par les concurrents, preuve d'une ambiance conviviale et d'une capacité à relativiser le classement.

31 La performance est donc définie prioritairement par rapport à soi et par rapport au groupe, c'est-à-dire en dehors d'une norme imposée de l'extérieur. Elle se négocie à l'intérieur de l'équipe, chacun étant attaché à donner le maximum pour contribuer au projet commun. Définie ainsi, la performance reflète des valeurs narcissiques, l'individu a rendez-vous avec lui-même pour une ré-appropriation du corps vécu sans distanciation normée. Toutefois, l'importance accordée au classement comme expression de la performance collective, montre que subsiste le besoin de comparaison aux autres, même si elle n'est pas quantitativement représentative dans l'énonciation des objectifs par rapport à l'équipe.

En effet, les réponses concernant ceux-ci évoquent massivement la convivialité, l'entraide, la participation, avec la volonté de " rester en groupe », « d'être une équipe soudée », de "mettre l'ambiance » et de "s'amuser». Le mode de recrutement est en relation directe avec la cohésion du groupe. De la cooptation entre amis de longue date, à la présence "par hasard » ou parce que « quelqu'un s'est désisté »: plus les liens sont étroits, plus l'équipe est soudée et créatrice d'une identité. Celle-ci se fonde dans un cas autour d'une ville étape : Gignac, rassemblant des personnes très proches du maire et très impliquées localement. La fierté de représenter la ville, la volonté de l'honorer et de faire parler d'elle est très prégnante dans leurs discours. Dans l'autre cas, c'est la défense d'une réputation (celle des Bretons, plus précisément des Rennois) qui sert de base identitaire : à savoir «faire la fête en mettant l'ambiance tous les soirs » et en «tenant sa place » sportivement parlant. La production d'une identité ne semble pas ici contradictoire à l'accomplissement de soi, ni source d'enjeux rivalitaires exacerbés, elle est au contraire tournée vers les autres.

L'analyse des relations aux autres équipes amène à faire une distinction entre les moments. Deux tendances divergent pendant les épreuves. Certains ont exactement les mêmes objectifs qu'au sein de leur propre équipe : créer des relations, s'entraider (" on a prêté une chambre à air ») et «ne pas se battre pour manger les autres »; quand d'autres jouent la rivalité. Ce rapport compétitif s'exprime surtout en course d'orientation pour une équipe proche, avec le déploiement de stratégies ( ne pas se faire voir ", « feinter ceux qui suivent ») qui pimente l'aventure ${ }^{8}$.

Hors-épreuves, toutes les équipes souhaitent rencontre les autres, chanter, s'amuser, "faire abstraction du temps et du résultat ». Les équipes locales qui avaient logé chez elles ou chez des amis, expriment leur désir de «s'intégrer " pour les autres soirées : la volonté participative est très forte.

Les relations à l'organisation sont aussi une dimension de l'être ensemble. Il semble que les attentes soient en adéquation avec ce que l'organisation met en place tant sur le plan formel (beaucoup d'éloges sur la qualité de l'organisation sportive et les règles imposées: mixité, déguisement) que sur le plan relationnel. Elle assure en quelque sorte une gestion du plaisir (dispensant les participants des soucis matériels ou sécuritaires) et une gestion du ludique (ce genre d'épreuve ne produit rien, la gratuité est souvent évoquée). Ces dimensions peuvent être considérées comme subversives par rapport à la logique sportive, car mettant en avant la spontanéité, le plaisir immédiat dans une pratique non productive.

La fête 

moment-clé. Les participants ont effectivement tous joué le jeu. L'appréciation est unanime: "c'est une idée géniale ", «super-sympa». Faire preuve d'imagination, d'originalité, «trouver une idée » est ressenti comme dynamisant par le groupe. Le déguisement est bien assimilé au registre de la fête, du ludique, du rire («les grands s'amusent »). La distance au sport est prise "ça enlève le côté Adidas » avec « moins de compétition, plus de jeu et de convivialité ». Deux contraintes seulement sont évoquées : trouver de la place dans les bagages, ou encore le risque de handicap avec la pratique sportive ${ }^{9}$. Si certains ont hésité ou n'avait pas encore prévu leur déguisement la veille, d'autres se sont "investis dès le départ en le peaufinant ». Dans ce cas, le déguisement renforce encore la cohésion de l'équipe au travers de son identité et de l'image qu'elle souhaite donner et se donner. Il est aussi vécu comme un changement dans le rapport avec les autres équipes, « une autre lien », " chaque équipe va rire en regardant les autres et réciproquement "; c'est un moyen de «finir sur une bonne impression ». Ainsi, la fête dans les épreuves est perçue dans la relation à l'équipe, le fait d'être en groupe, et ce particulièrement dans le moment fort du déguisement qui clôturait le raid en signant sa symbolique (le passage de l'embouchure). Ce moment traduit la dimension transgressive de cette «fête des corps ", détournement jusqu’à la dérision des normes productives et comparatives du sport. avec deux explications différentes. Il y a ceux qui veulent participer mais qui sont "vraiment morts le soir». Tous ne peuvent pas associer sport et fête et si le choix s'impose, la fête nocturne est sacrifiée (signifiant pour ceux-ci la prévalence de la norme sportive). Et il y a les déçus "par le manque de monde», par le côté «moins chaleureux des repas par rapport aux épreuves » ou encore la frustration de ceux qui ont voulu mettre l'ambiance mais "n'ont pas été suivi», ressentant les autres équipes comme « à l'écart ».

L'observation, l'attention « au sensible » conforte et complètent l'analyse, montrant les différentes formes prises par la fête, qui ne peut se circonscrire à une définition close (les repas, les soirées.....) et qui n'est pas forcément là où l'attendait l'organisation (et même peut-être les participants).

39 En permanence, du début à la fin du raid, celle qui est première, c'est «la fête de l'équipe, de l'être ensemble " (ce que décrivent les paragraphes précédents). Dans la confrontation à soi-même et aux autres, l'individu choisit ses pairs pour une pratique « tribale » : réunion ponctuelle d'une équipe partageant le même objectif, le même style de vie, et sacrifiant à la même passion. Le groupe est solidaire, mais aussi solitaire, en autonomie dans un milieu inconnu, tout en se mesurant à d'autres, qui font la même chose, au même moment (un mélange de communion et de rivalité). C'est l'illustration d'un «lien social émotionnel » (MAFFESOLI, 1990), où la pratique conviviale ménage une forme de relation surprenante: le "vivre ensemble séparément ». Une relation intimiste, à quelques uns, pour un partage intense mais éphémère; d'où peut-être la déception de quelques uns dans le passage au grand groupe, lorsque la célébration collective ne prend pas le relais de la relation intimiste de l'équipe (ou perd en qualité et en intensité).

Mais la fête prend aussi des formes différentes au cours du raid. Les deux premières journées sont plutôt celles de la «la fête de la nature ». Il se dégage une ambiance conviviale sur fond de verdure ou de hameau paisible que vont égayer les concurrents 
par leur flot coloré accompagné d'une sonorisation entre rock et reggae. C'est aussi « la fête de la rivière » quand au petit matin, dans un cadre magnifique, se mélangent dans les reflets de l'eau troublés par les premières éclaboussures, les couleurs de la nature et celles des canoës jaunes et des gilets de sauvetage violets. Images qui se marient à un panachage sonore insolite: un coup de sifflet toutes les trois minutes, le chant des oiseaux, les éclats de rires, le clapotis de l'eau et ....l'hélico. A la fin de ces deux journées, les repas permettent tout juste à quelques chants de pointer : la fatigue et les conditions matérielles portent une grande part de responsabilité ${ }^{10}$. Les soirées n'attirent effectivement pas les participants et seules quelques équipes poursuivront tardivement la fête à leur façon.

41 La troisième journée prend les allures de « la fête effervescente ». Le ton est donné avec l'épreuve déguisée : les équipes traversent le vieux Agde en chantant pour le plus grand plaisir des touristes curieux. Elles donnent à voir une troupe bigarrée, aux effluves composites, associant à l'iode marin l'odeur du maquillage et des bombes colorantes, sur fond d'animation de plage et de friture, avec bien sûr toujours...... les rotations de l'hélicoptère. A n'en pas douter, nul besoin de terres étrangères pour vivre l'exotisme : juxtaposition des contraires, ambiance esthétique et polysensuelle, le "processus de melting-pot " (LIPOVETSKY, 1983) participe à la fête, exprimant " un hédonisme du quotidien irrépressible et puissant » (MAFFESOLI, 1990).

42 Le soir, la fête éclate, plus chaleureuse que délirante : l'intensité du lien social relève encore davantage du petit groupe. Tout le monde se connait bien après avoir vécu ensemble une tranche de vie éphémère. Les photos exposées sont les preuves tangibles de moments partagés qui suscitent commentaires et rires, et qui seront bientôt de l'ordre du souvenir.

43 La satisfaction exprimée globalement par les participants montre que les organisations sont capables d'être à l'écoute et de se transformer pour promouvoir des pratiques ludiques et sportives allant dans le sens d'une adéquation à la demande. Le Raid Passion Hérault est "une autre façon de pratiquer" où cohabite la permanence anthropologique du sport au sens de l'épreuve (s'éprouver), de se mesurer (à soi-même, aux autres) et celle d'être en groupe, de partager. Le changement des normes, des lieux, des mises en scène permet l'expression des valeurs narcissiques de personnalisation, d'esthétisme et d'hédonisme et le renouvellement des formes « d'être ensemble ». La performance reste prégnante tout en s'exprimant différemment : c'est un sport horsnormes... sur fond de «culture performative " (LE POGAM, Y., 1998). C'est pourquoi il faut être prudent dans l'affirmation de distanciation au modèle sportif compétitif, d'autant plus que l'évolution de ces épreuves montre leur sportivisation, pendant que la nature qui leur sert de cadre est domptée et rentabilisée ${ }^{11}$.

A travers cet événement se lit un lien social comme expression de soi et de l'être ensemble: plaisir de l'investissement dans de nouvelles pratiques («l'aventure »), plaisir de se retrouver face à soi-même pour s'éprouver mais au travers d'un projet commun plus ou moins élaboré et négocié avec une gestion collective des ressources et la valorisation des relations conviviales. Le lien social se lit également comme appropriation de l'événement par les participants permettant leur créativité. Les épreuves en général (le déguisement en particulier) en ont été des moments forts. Les équipes l'ont traduit à travers des stratégies plurielles renvoyant elles-mêmes à des imaginaires diversifiés attestant de cette personnalisation collective (à chaque équipe 
son projet, a chacun son aventure): témoignage de la capacité du groupe à s'autonomiser.

Sans perdre de vue le caractère limité et ponctuel de cette expérimentation, il semble possible de la restituer dans le débat de l'autodétermination de l'individu ou de son conditionnement par le système marchand (ou encore dans l'alternative d'un individualisme hédonisme utilitariste ou souverain, LE POGAM, Y., 1997).

Il n'est pas question d'ignorer ici la logique économique qui régit (aussi) les épreuves de sport-aventure: elles servent des objectifs de promotion d'une entreprise, d'une région. La fête, le déguisement peuvent être des arguments marketing et servir une logique médiatique à l'affût de spectaculaire ${ }^{12}$. La reconduction de l'événement irait certainement dans le sens de son intégration dans la logique sportive et managériale. Mais le débat dépasse la nature des activités sportives (canoë, course à pied....) ou le registre dans lequel elles s'inscrivent (pratiques "fun", sport-aventure...). Il met en cause la possibilité pour l'individu de s'exprimer dans des pratiques (fussent-elles corporelles, sportives voire organisées) de manière expressive, ludique, improductive qui ne renvoient donc pas uniquement à un corps normé par la productivité ou aliéné par la consommation.

La faveur a été donné ici à la fois au regard phénoménologique, au « sens des sens », et aux théories individualistes qui, d'un point de vue épistémologique, mettent l'accent sur l'autonomie et la responsabilisation de l'individu. L'hypothèse serait ici d'une possible cohabitation de logiques posées comme contraires, postulant que l'individu peut être consommateur tout en gardant une capacité d'autodétermination. C'est le sens que les acteurs donnent à leurs pratiques qui renouvellent les formes de leur engagement, et par là même porte les germes du changement. Le corps a un rôle central, «un statut d'opérateur sociétal» (BERTHELOT, J.M., 1983, p.121); non seulement il incorpore le social, mais il est aussi émetteur, producteur de social. Les formes (ici du lien social, de la fête) mettent en évidence le point d'interface entre l'ordre des structures et celui des acteurs. A l'intérieur de l'équipe se côtoient des moments de partage, d'échange gratuit, ludique, en même temps que se construit un projet pour une performance classée et référée par rapport aux autres. Le participant s'inscrit dans une logique de consommation, il profite de l'organisation qui lui donne les moyens de participer, de "s'éclater », ce qui n'est pas forcément contradictoire avec son autodétermination grâce à " l'espace de jeu " $13^{13}$ ménagés par l'organisation. Dans le Raid Passion Hérault, cette autonomie s'est repérée notamment vis à vis de la fête, qui reste ce qu'il en fait, quand et où il le souhaite, dans l'ici et maintenant de la relation à l'autre ou à la nature. Il y a dans la fête une immanence, une spontanéité qui ne relèvent pas de l'organisé, elle reste subtile, insaisissable, de l'ordre du désordre....et ne se fait pas forcément là où elle est prévue et attendue.

Jouer sur le temps, l'espace, c'est aussi modifier les codes qui remanient les formes de rapports (réels et symboliques) que l'homme entretient avec la société. Les modalités d'organisation ont une influence non négligeable sur la signification des pratiques et la marge de liberté offerte fonctionne à différents niveaux : dans la réalité des conditions de l'épreuve, mais aussi dans l'imaginaire, les représentations, donnant une chance à chacun de trouver ce qu'il est venu chercher. En favorisant l'expression de soi, l'appropriation de l'événement par l'individu, ces modalités peuvent permettre l'expression d'un lien social renouvelé et intégrateur mais susceptible d'être capturé par la logique rentabiliste. 


\section{BIBLIOGRAPHIE}

DAVISSE, A., LOUVEAU, C., 1991, Sports, école, société : la part des femmes, Paris, Actio.

LACROIX, G., 1991, La performance : une valeur renouvelée, in Actes du Colloque Performance et Santé, Université de Nice, UFRSTAPS, Sophia-Antipolis, Co-édition AFRAPS et LANTAPS.

LACROIX, G., 1993, Le sport-aventure, une forme d'innovation sportive, in Sport et Management, ouvrage collectif sous la direction d'A. LORET, Dunod, pp 171 - 182.

LE BRETON, David, 1991, La passion du risque, Métailié.

LE POGAM, Y., 1995, L'anthropo-sociologie de Pierre Sansot : le sport et le sensible, Revue Corps et Culture, 1, Montpellier, pp 102 - 138.

LE POGAM, Y., 1997, Corporéisme et individualisme hédoniste, Revue Corps et Culture, 2, Montpellier, pp 161 - 169

LE POGAM, Y., 1998, (sous presse), Sport, modernité et souveraineté, Les Cahiers de l'Imaginaire, 16, Centre de Recherche sur l'Imaginaire (CRI), Université Paul Valéry, Montpellier, Toulouse, Privat LIPOVETSKY, G., 1983, L'ère du vide : essais sur l'individualisme contemporain, Gallimard.

MAFFESOLI, M., 1988, Le temps des tribus, Librairie des Méridiens, Klincksieck et Cie.

MAFFESOLI, M., 1990, Au creux des apparences, pour une éthique de l'esthétique, Plon.

VIGARELLO, G., 1982, Les vertiges de l'intime, Le laboratoire des sciences humaines in ESPRIT, Le corps.... entre illusions et savoirs, Revue mensuelle, 2, Février, pp. 68-90.

YONNET, P., 1985, Jeux, modes et masses (Chap. 2, Joggers et marathoniens), Gallimard, pp 91 - 131.

\section{ANNEXES}

\section{Notes méthodologiques}

(Ont participé à la préparation de cette recherche Y. LE POGAM et Nathalie LEFEVRE, nous remercions particulièrement cette dernière qui a effectué tous les interviews et recueilli les données sur l'ambiance de la manifestation.)

L'analyse structurelle situe les caractéristiques de l'événement (première partie), les interviews sondent le vécu des participants, les observations extérieure et participante complètent le recueil de données par des informations sur l'ambiance et le type de communication.

Dans la grille d'interview, des variables situent la sportivité des personnes (pratiques, fréquence) et leur objectif individuel ; la modalité de constitution des équipes, les objectifs qu'elle se donne pour elle-même et par rapport aux autres.

D'autres variables sondent le vécu de l'épreuve déguisée, de la mixité; l'investissement hors-épreuves ; les attentes par rapport à l'organisation ; les moments forts et la signification accordée aux termes : « le sport, l'aventure, la fête ». Elles permettent de recueillir des éléments sur la réalité de la " cassure » avec le modèle sportif compétitif, sur le vécu de la fête pendant et hors-épreuves, sur la socialité à l'oeuvre et les images associées au Raid Passion Hérault. 
L'échantillon de la population a été construit en croisant 4 variables rendant compte de la population mère : l'expérience de ce type d'épreuves (équipes nouvelles ou anciennes); la moyenne d'âge (jeunes et moins jeunes) ; les objectifs (gagner ou participer); et l'origine géographique (départementales ou nationales).

La durée des interviews est variable, les plus courts étant de 20 minutes. Toutes les personnes de la même équipe sont interrogées mais le moment peut varier $: 2^{\mathrm{e}}$ ou $3^{\mathrm{e}}$ raid. Les interviews de quatre équipes (sur 22) ont été recueillies.

Les limites de la méthodologie tiennent d'une part à la position de l'observatrice rédactrice qui était également la conceptrice et organisatrice du raid ; d'autre part à la faible quantité d'interview exploitables (18 sur 132 participants).

\section{NOTES}

1. Pour P. Yonnet : « l'aventure, c'est faire ce qui n'a pas été fait (l'inconnu absolu) ou ce que l'on n'a pas fait (l'inconnu relatif) ». Dans le premier cas « est posée la question de l'adaptation d'un individu à un environnement qu'il s'agit d'explorer dans des circonstances inconnues »; dans le deuxième cas, l'aventure de masse «c'est l'individu lui-même qui devient le théâtre premier de l'exploration » et la question posée est inverse : « comment un individu mené au-delà de ses limites ordinaires va-t-il s'adapter à un environnement et des circonstances déjà définies? ». "L'inconnu relatif » prend le pas sur «l'inconnu absolu ». YONNET, P., 1989, La culture du vertige, in Dossier Spécial La folie de l'extrême, Le nouvel observateur, 12 - 18 Janvier

2. Ceci renvoie aux travaux de recherches de la conceptrice et organisatrice sur l'implantation, le développement des sports fun et d'autres pratiques dites «nouvelles » (courses sur route et pratiques de mise en forme), ainsi que sur les épreuves de sport-aventure (G. LACROIX,1984,1991,1993).

3. L'organisation se heurte ici aux représentations traditionnelles des partenaires (collectivités territoriales, entreprises privées) pour lesquels le résultat sportif reste le seul critère du classement final, proclamé dans la tradition protocolaire.

4. Cf. note 2

5. Cf. note 1

6. La « débrouille » fréquemment évoquée, renvoie à des représentations très actuelles de l'aventure, avec sous-jacente celle de l'efficacité du petit groupe, mobile, rusé. (Les " schèmes de la guérilla », POCIELLO, Ch., 1987, Revue Esprit,4).

7. Ceci est peut-être aussi lié à la représentation qu'ont les concurrents de l'attente des médias.

8. Cf. note 6

9. Cette inquiétude est justifiée : le relais en course à pied et canoë se déroule à Agde de 15 à 16 heures, sous un grand soleil et l'équipe est déguisée en plongeurs (combinaison néoprène intégrale, masque, tuba.......).

10. Le premier soir, la salle de restaurant est trop petite et les voûtes, les colonnes empêchent la communion collective. Le deuxième soir, le repas frise la catastrophe par l'incapacité du restaurateur à fournir quantitativement et qualitativement une prestation acceptable!

11. L'évolution actuelle prouve que les épreuves de sport-aventure sont entrées dans une logique compétitive équivalente aux sports de haut-niveau dans sa forme 
institutionnalisée. Même dans des épreuves locales sans importance ni enjeux particuliers, le changement d'un ou deux paramètres (notamment les récompenses aux vainqueurs) fait totalement basculer la logique des relations, de conviviales à rivalitaires.

12. Toutefois, le Raid Passion Hérault n'est pas représentatif de l'événementiel au sens économique et médiatique, l'organisation et l'encadrement sont bénévoles dans le cadre d'une association à but non lucratif.

13. « Ensemble de choix au sein duquel, selon leur positionnement à un moment donné, les divers acteurs élaborent, en fonction des choix objectifs proposés, les stratégies que leur suggèrent les enjeux révélés » (...) « La liberté de l'acteur s'inscrit alors non seulement dans la définition de la meilleure stratégie possible, mais aussi dans l'essai de saisie de plus en plus aiguë des règles du jeu, et au-delà dans la tentative d'action concertée pour modifier les règles, voire transformer de fond en comble le jeu luimême ». BERTHELOT, J.M., 1983, Le piège scolaire, p. 221.

\section{RÉSUMÉS}

Les épreuves de sport-aventure, nées dans les années 80, remportent un franc succès et se multiplient. Elles associent différentes activités sportives, se déroulent en pleine nature et se courent par équipe. L'objectif est de dégager les caractéristiques et les composantes du lien social, en fonction du type d'organisation et d'épreuves. Dans l'événement pris en exemple ici, l'hypothèse postule que le mode de sociabilité est fonde sur la convivialité, le partage et la dimension festive par la mise à distance de certaines caractéristiques du sport traditionnel. La mise en rapport de l'offre faite dans la production de l'événement avec le vécu des participants tente de qualifier les formes d'être ensemble, par un regard qui se veut attentif « au sensible ».

From the eighties, the sport-adventure events are successful and grow in number. These outdoor events concern different sport activities and run by team. The aim is to release characteristics and components of the social bond related to the organisation and to the competition. In this example, the hypothesis postulates that the mode of sociability is more based on social interaction and sharing by standing aloof some sport and by festive dimensions. The connexion between the offer included in the event and the actual experience of the participants describes the different types of sociability by a methodological approach of the "sensitive".

\section{INDEX}

Mots-clés : imaginaire, événement, fête, " être ensemble »

Keywords : event, representation, feast, sociability 
AUTEUR

GISĖLE LACROIX

Faculté des Sciences du Sport et de l'Education Physique. Université Montpellier 1.

Equipe "Corps et Culture" 